\title{
Scalar Singularly Perturbed Cauchy Problem for a Differential Equation of Fractional Order
}

\author{
Burkhan Kalimbetov \\ Department of Mathematics Akhmed Yasawi University \\ Turkestan, Kazakhstan, \\ E-mail: burkhan.kalimbetov [AT] ayu.edu.kz.
}

\begin{abstract}
In this paper we consider initial problem for an ordinary differential equation of fractional order with a small parameter for the derivative. S.A. Lomov regularization method is used to construct an asymptotic approximate solution of the problem with accuracy up to any power of a small parameter. Using the computer mathematics system (CMS) Maple, a symbolic solution of the original problem is obtained, and solution schedules are constructed, depending on the initial data and various values of the small parameter. It is shown that the asymptotic solution presented in the form of a specific convergent series and the solution represented by the CMS Maple coincides with the exact solution of the original problem.
\end{abstract}

Keywords--- singular perturbation, asymptotic solution, regularization of the problem, iterative problem, computer mathematics system Maple.

\section{INTRODUCTION}

In recent years, interest has increased in study of so-called differential equations of fractional order, in which the unknown function is contained under the sign of the derivative of fractional order. This is due both to development of the theory of fractional integration and differentiation, and to applications of such structures in various fields of science [1].

In applied theory, a special place is occupied by methods of asymptotic integration of differential equations. Despite the rapid growth of computational methods, developed to solve applied problems, the need for asymptotic analysis continues unabated. Even in cases where solutions of equations are known, such analysis provides an invaluable service in their physical interpretation.

In the paper we consider the initial problem for a singularly perturbed differential equation of fractional order:

$$
L_{\varepsilon} y(t, \varepsilon) \equiv \varepsilon y^{(1 / 2)}+y=t, \quad y(0, \varepsilon)=y^{0}, \quad t \in[0, T], \quad T>0,
$$

where $\varepsilon>0$ a small parameter, $y^{0}$ a constant number. It is required to find an asymptotic solution of the problem (1) as $\varepsilon \rightarrow+0$.

By definition of the fractional derivative [1,2], derivative $y^{(1 / 2)}$ is denoted as $\sqrt{t} \frac{d y(t, \varepsilon)}{d t}$. Then the problem (1) has the following form:

$$
L_{\varepsilon} y(t, \varepsilon) \equiv \varepsilon \sqrt{t} \frac{d y}{d t}+y=t, \quad y(0, \varepsilon)=y^{0} .
$$

Solution of the homogeneous equation of the problem (2) is a function, $y_{0}(t, \varepsilon)=C e^{-\frac{2}{\varepsilon} \sqrt{t}}$, where $C$ an arbitrary constant, and solution of the problem (2) is

$$
y(t, \varepsilon)=\left(y^{0}-\frac{\varepsilon^{2}}{2}\right) e^{-\frac{2}{\varepsilon} \sqrt{t}}+t-\varepsilon \sqrt{t}+\frac{\varepsilon^{2}}{2} .
$$

\section{PROBLEM REGULARIZATION}

We introduce a regularizing variable [3]:

$$
\tau=\frac{1}{\varepsilon} \int_{0}^{t} \frac{d t}{\sqrt{t}}=-\frac{2}{\varepsilon} \sqrt{t} \equiv \varphi(t) / \varepsilon
$$

and instead of the desired solution $y(t, \varepsilon)$ of the problem (2), we will study some extended function $\tilde{y}(t, \tau, \varepsilon)$. From the extended function, we require that the following identity be satisfied: 


$$
\left.\tilde{y}(t, \tau, \varepsilon)\right|_{\tau=\phi(t, \varepsilon)} \equiv y(t, \varepsilon) .
$$

For the extended function $\tilde{y}(t, \tau, \varepsilon)$ the following "extended" problem corresponds:

$$
\tilde{L}_{\varepsilon} \tilde{y}(t, \tau, \varepsilon) \equiv \varepsilon \sqrt{t} \frac{\partial \tilde{y}}{\partial t}-\frac{\partial \tilde{y}}{\partial \tau}+\tilde{y}=t, \quad \tilde{y}(0,0, \varepsilon)=y^{0} .
$$

The main advantage of the problem (4) over the problem (2) is that its solution $\tilde{y}(t, \tau, \varepsilon)$ can be searched in the form of a regular classical series in powers of $\varepsilon$ :

$$
\tilde{y}(t, \tau, \varepsilon)=\sum_{k=0}^{\infty} \varepsilon^{k} y_{k}(t, \tau)
$$

where $y_{k}(t, \tau) \in C^{\infty}[0, T]$

Putting the series (5) intothe «extended» problem (4), and equating coefficients of the same powers of $\varepsilon$, we get the following iteration problems:

$$
\begin{aligned}
& L_{0} y_{0}(t, \tau) \equiv-\frac{\partial y_{0}}{\partial \tau}+y_{0}=t, \quad y_{0}(0,0)=y^{0} ; \\
& L_{0} y_{1}(t, \tau)=-\sqrt{t} \frac{\partial y_{0}}{\partial t}, \quad y_{1}(0,0)=0 \\
& L_{0} y_{2}(t, \tau)=-\sqrt{t} \frac{\partial y_{1}}{\partial t}, \quad y_{2}(0,0)=0 ; \\
& \cdots \\
& L_{0} y_{k}(t, \tau)=-\sqrt{t} \frac{\partial y_{k-1}}{\partial t}, \quad y_{k}(0,0)=0 ;
\end{aligned}
$$

\section{SOLVABILITY OF ITERATION PROBLEMS}

To solve the equation (6), we consider the variable $t$ as a parameter, i.e.

$$
\frac{\partial y_{0}}{y_{0}-t}=\partial \tau, \quad \ln \left|y_{0}-t\right|=\tau+\ln C, \quad y_{0}(t, \tau)=C e^{\tau}+t .
$$

Due to the initial condition $y_{0}\left(0,0=y^{0}\right.$, we define a solution of the problem (6) in the form:

$$
y_{0}(t, \tau)=y^{0} e^{\tau}+t
$$

We turn to the next iteration problem (7). Taking into account (10), it has the following form:

Solving this equation, as the equation (6), we obtain:

$$
L_{0} y_{1}(t, \tau) \equiv-\frac{\partial y_{1}}{\partial \tau}+y_{1}=-\sqrt{t}, \quad y_{1}(0,0=0 .
$$

$$
y_{1}(t, \tau)=C e^{\tau}-\sqrt{t} .
$$

Initial condition $y_{1}\left(0,0=0\right.$, allows us to determine the function $y_{1}(t, \tau)=-\sqrt{t}$. unambiguously. Putting this value of the function $y_{1}(t, \tau)$ into the equation of the problem (8), we get:

$$
L_{0} y_{2}(t, \tau) \equiv-\frac{\partial y_{2}}{\partial \tau}+y_{2}=\frac{1}{2}, \quad y_{2}(0,0=0 .
$$

Solution of the problem (8) is the function:

$$
y_{2}(t, \tau)=C e^{\tau}+\frac{1}{2}
$$

Taking into account the initial condition $y_{2}\left(0,0=0\right.$, we have $y_{2}(t, \tau)=\frac{1}{2}$.

Subsequent partial derivatives with respect to the variable $t$ are equal to zero, i.e. $\frac{\partial y_{3}}{\partial t} \equiv \ldots \equiv \frac{\partial y_{k}}{\partial t} \equiv \ldots \equiv 0$.

Putting the found solutions $y_{0}(t, \tau), y_{1}(t, \tau), \ldots, y_{k}(t, \tau), \ldots$ into the series (5), and making constriction $\left.\tilde{y}(t, \tau, \varepsilon)\right|_{\tau=\frac{\varphi(t)}{\varepsilon}} \equiv y(t, \varepsilon)$, we obtain:

$$
y(t, \varepsilon)=\left(y^{0}-\frac{\varepsilon^{2}}{2}\right) e^{-\frac{2}{\varepsilon} \sqrt{t}}+t-\varepsilon \sqrt{t}+\frac{\varepsilon^{2}}{2} .
$$


This shows that solutions (3) and (12) coincide.

\section{JUSTIFICATION OF SOLVABILITY OF ITERATION PROBLEMS}

We return to the iteration problems (6) - (9). Solutions of the problem (6) will be defined in the following form:

$$
y_{0}(t, \tau)=y_{0}^{(0)}(t)+y_{1}^{(0)}(t) e^{\tau},
$$

where $y_{j}^{(0)}(t) \in C^{\infty}[0, T], j=0,1$.

Putting (13) into (6), and equating coefficients of exponents and free members, we get

$$
\begin{aligned}
& 0 \cdot y_{1}^{(0)}(t)=0, \\
& y_{0}^{(0)}(t)=t
\end{aligned}
$$

where we find $y_{1}^{(0)}(t)$ arbitrary function, thus we defined solution of the equation (6) in the following form:

$$
y_{0}(t, \tau)=\alpha(t) e^{\tau}+t
$$

where $\alpha(t) \in C^{\infty}[0, T]$ while an arbitrary function. Subjecting (14) to the initial condition $y_{0}(0,0)=y^{0}$, we find $\alpha(0)=y^{0}$.

Let's turn to (7). Due to (14), equation (7) has the following form:

$$
-\frac{\partial y_{1}}{\partial \tau}+y_{1}=-\sqrt{t} \cdot \alpha^{\prime}(t) e^{\tau}-\sqrt{t}
$$

We look for its solution as

$$
y_{1}(t, \tau)=y_{0}^{(1)}(t)+y_{1}^{(1)}(t) e^{\tau}
$$

Putting (15) into the equation, and equating coefficients of exponents and free members, we get

$$
\begin{aligned}
& 0 \cdot y_{1}^{(1)}(t)=-\sqrt{t} \cdot \alpha^{\prime}(t), \\
& y_{0}^{(1)}(t)=-\sqrt{t},
\end{aligned}
$$

For solvability of the first equation it is necessary and sufficient that $\alpha^{\prime}(t)=0$. Taking into account $\alpha(0)=y^{0}$, we find $\alpha(t)=y^{0}$ un ambiguity.

Therefore, we have found the solution (13) of the first iteration problem (6) in the following form:

$$
y_{0}(t, \tau)=y^{0} e^{\tau}+t .
$$

In its turn, solution (15) of the second iteration problemis defined in the form:

$$
y_{0}(t, \tau)=\beta(t) e^{\tau}-\sqrt{t},
$$

where $\beta(t) \in C^{\infty}[0, T]$ while an arbitrary function.

In study solvability of the problem (8), a value of the function $\beta(t)$, is unambiguously determined, thereby, approximations of higher orders are calculated.

We formulate the corresponding result in the form of the following proposition.

Theorem. During consistent solution all iteration problems (6) - (9) are uniquely solvable in the class of functions $y_{k}(t, \tau)=y_{0}^{(k)}(t)+y_{1}^{(k)}(t) e^{\tau}, \quad y_{j}^{(k)}(t) \in C^{\infty}[0, T], j=0,1$.

\section{SYMBOLIC AND NUMERICAL ANALYSIS OF THE RESULTS}

Now we will find a solution to the problem (2) by means of the Maple computer mathematics system [4]:

> restart; with (plots); ode :=epsilon*sqrt $(t) * \operatorname{diff}(y(t), t)=-y(t)+t$;

>ics $:=\mathrm{y}(0)=\mathrm{A}$;

$$
\text { ode }:=\varepsilon \sqrt{t}\left(\frac{\partial}{\partial t} \mathrm{y}(t)\right)=-\mathrm{y}(t)+t
$$

$$
\text { ics }:=\mathrm{y}(0)=A
$$

> dsolve ([ode, ics]);

$$
\mathrm{y}(t)=t-\varepsilon \sqrt{t}+\frac{1}{2} \varepsilon^{2}+\mathbf{e}^{\left(-2 \frac{\sqrt{t}}{\varepsilon}\right)}\left(-\frac{1}{2} \varepsilon^{2}+A\right)
$$




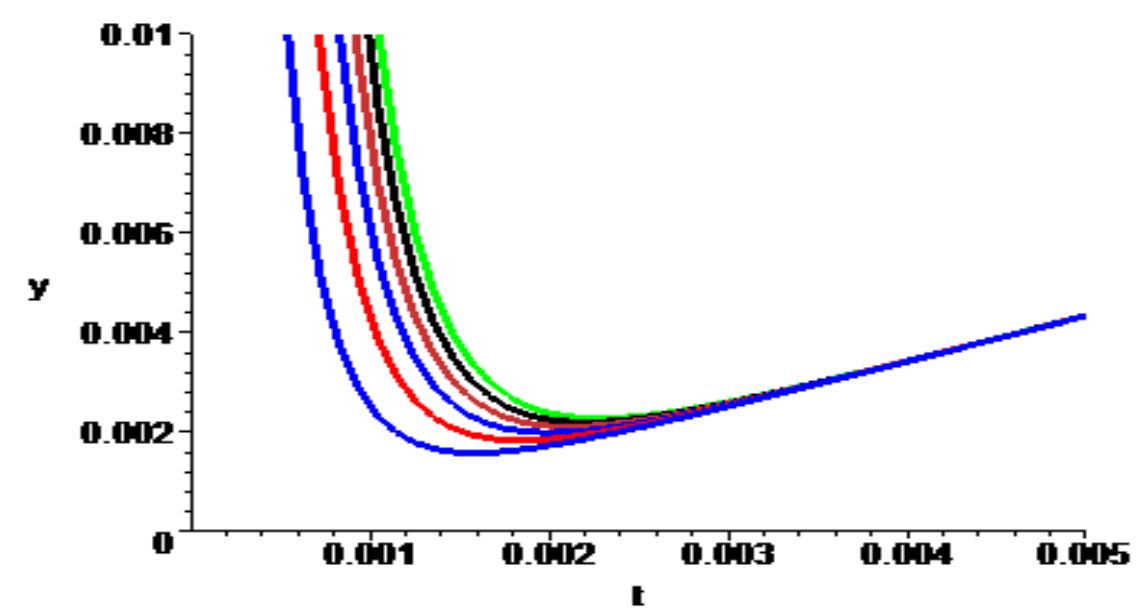

Different values of these solutions depending on the values of the small parameter $\varepsilon$ and the constant $y^{0}$ are given in the following table:

\begin{tabular}{|c|c|c|c|c|c|c|}
\hline & $t=0$ & $t=0,2$ & $t=0,4$ & $t=0,6$ & $t=0,8$ & $t=1$ \\
\hline$\varepsilon=0.010$ & 0 & 0,1955778 & 0,393725 & 0,592304 & 0,791105 & 0,99005 \\
\hline$\varepsilon=0,025$ & 0 & 0,189132 & 0,3845 & 0,58094 & 0,77795 & 0,975312 \\
\hline$\varepsilon=0,050$ & 0 & 0,17888 & 0,36962 & 0,56252 & 0,75652 & 0,95125 \\
\hline$\varepsilon=0,075$ & 0 & 0,16927 & 0,355378 & 0,544717 & 0,73573 & 0,927812 \\
\hline$\varepsilon=0,100$ & 0 & 0,1602779 & 0,34175 & 0,52754 & 0,71555 & 0,905 \\
\hline
\end{tabular}

Acknowledgments. This work is supported by the grant AP05133858 "Contrast structures in singularly perturbed equations and their application in the theory of phase transitions" by the Scientific Committee of the Ministry of Education and Science of the Republic of Kazakhstan.

\section{REFERENCES}

[1] Khalil, R., Al Horani, M., Yousef, A. and Sababheh, M. (2014) A new definition of fractional derivative, Journal Comput. Appl. Math., 264, 65-70.

[2] Katugampola, U. (2015) Correction to "What is a fractional derivative?" by Ortigueira and Machado. Journal Computational Physics, 293, 4-13.

[3] Lomov, S.A. (1992) Introduction to General Theory of Singular Perturbations, 112, American Mathematical Society, Providence, USA.

[4] Kirsanov, M.N. (2007) Counts of in Maple, M, Fizmatlit. 